\title{
Initiation of metamorphosis and control of ecdysteroid biosynthesis in insects: The interplay of absence of Juvenile hormone, PTTH, and $\mathrm{Ca}^{2+}$-homeostasis
}

\author{
Arnold De Loof ${ }^{\mathrm{a}, *}$, Tim Vandersmissen ${ }^{\mathrm{b}}$, Elisabeth Marchal ${ }^{\mathrm{c}}$, Liliane Schoofs ${ }^{\mathrm{a}}$ \\ a Functional Genomics and Proteomics Group, Department of Biology, KU Leuven-University of Leuven, Belgium \\ b Department of Teacher Education, Leuven University College, Leuven, Belgium \\ ${ }^{\mathrm{c}}$ Molecular Developmental Physiology and Signal Transduction Group, Department of Biology, KU Leuven-University of Leuven, Belgium
}

\section{A R T I C L E I N F O}

\section{Article history:}

Received 23 May 2014

Received in revised form 25 July 2014

Accepted 25 July 2014

Available online $\mathrm{xxx}$

\section{Keywords:}

Insect hormones

Torso

Apoptosis

PTTH

Prothoracic glands

Thymus

\begin{abstract}
A B S T R A C T
The paradigm saying that release of the brain neuropeptide big prothoracicotropic hormone (PTTH) initiates metamorphosis by activating the Torso-receptor/ERK pathway in larval prothoracic glands (PGs) is widely accepted nowadays. Upon ligand-receptor interaction $\mathrm{Ca}^{2+}$ enters the PG cells and acts as a secondary messenger. Ecdysteroidogenesis results, later followed by apoptosis. Yet, some data do not fit in this model. In some species decapitated animals can still molt, even repeatedly, and metamorphose. PTTH does not universally occur in all insect species. PGs may also have other functions; PGs as counterpart of the vertebrate thymus? There are also small PTTHs. Finally, PTTH remains abundantly present in adults and plays a role in control of ecdysteroidogenesis (=sex steroid production) in gonads. This is currently documented only in males. This urges a rethinking of the PTTH-PG paradigm. The key question is: Why does PTTH-induced $\mathrm{Ca}^{2+}$ entry only result in ecdysteroidogenesis and apoptosis in specific cells/tissues, namely the PGs and gonads? Indeed, numerous other neuropeptides also use $\mathrm{Ca}^{2+}$ as secondary messenger. The recent rediscovery that in both invertebrates and vertebrates at least some isoforms of $\mathrm{Ca}^{2+}$-ATPase need the presence of an endogenous farnesol/juvenile hormone(JH)-like sesquiterpenoid for keeping cytosolic $\left[\mathrm{Ca}^{2+}\right]_{i}$ below the limit of apoptosis-induction, triggered the idea that it is not primarily PTTH, but rather the drop to zero of the JH titer that acts as the primordial initiator of metamorphosis by increasing $\left[\mathrm{Ca}^{2+}\right]_{i}$. PTTH likely potentiates this effect but only in cells expressing Torso. PTTH: an evolutionarily ancient gonadotropin?
\end{abstract}

(C) 2014 Elsevier Inc. All rights reserved.

\section{Introduction}

The isolation in 1954 of $25 \mathrm{mg}$ of pure material with moltingand metamorphosis hormone activity, later named ecdysone, from about $500 \mathrm{~kg}$ of pupae of the silkworm Bombyx mori by Butenandt and Karlson, followed in 1965 by the identification of its steroid

Abbreviations: CA, corpus allatum; DH, diapause hormone; E and 20E, ecdysone and 20-hydroxyecdysone; ERK, extracellular signal-regulated kinase; JH, juvenile hormone; PG, prothoracic gland; PTTH, prothoracicotropic hormone; RER and SER, rough and smooth endoplasmic reticulum.

* Corresponding author at: Functional Genomics and Proteomics Group, Department of Biology, KU Leuven-University of Leuven, Naamsestraat 59, 3000 Leuven, Belgium. Tel.: +32 16324260 .

E-mail addresses: arnold.deloof@bio.kuleuven.be (A. De Loof), tim.vandersmissen@khleuven.be (T. Vandersmissen),

Elisabeth.marchal@bio.kuleuven.be (E. Marchal), liliane.schoofs@bio.kuleuven.be (L. Schoofs).

http://dx.doi.org/10.1016/j.peptides.2014.07.025

0196-9781/@ 2014 Elsevier Inc. All rights reserved. chemical nature, was a true landmark in insect endocrinology. This story has been summarized by Karlson [36]. When in 1967 Röller and Dahm isolated enough material with juvenile hormone $(\mathrm{JH})$ activity from the male accessory glands of another silkworm, namely Hyalophora cecropia and identified it as an ester of the sesquiterpenoid farnesol, the way was open for fast progress in invertebrate endocrinology. A historical account of this discovery can be found in [55]. The searches for the (brain) secretory factors controlling the biosynthesis of these two key hormones also count as true landmarks. In a heroic effort that started in 1960 lasting a few decades, Japanese research teams succeeded in isolating, from a total of more than $2.10^{7}$ Bombyx heads (historical account in Ishizaki and Suzuki [32]), two proteinaceous factors, named Prothoracicotropic hormone (or big PTTH) and bombyxin (or small PTTH). These hormones initiated further development in decapitated pupae of another moth, namely Samia cynthia, as well as the release of ecdysteroids from isolated prothoracic glands (PGs). The identification of the peptide structure of brain hormones 
that either inhibit (=allatostatins) or stimulate (=allatotropins) $\mathrm{JH}$ biosynthesis by the corpora allata (CA) in an in vitro radiochemical assay, originally developed by Pratt and Tobe [53], also substantially broadened the field of invertebrate endocrinology [68].

Yet, several questions remain unanswered; in particular with respect to the different putative sites of ecdysteroid biosynthesis, the existence of other small PTTHs, the possible role of PTTH in functions other than molting and metamorphosis, the speciesspecificity of PTTH, the function of apoptosis in the PGs, and last but not least, the role of $\mathrm{JH}$. This paper focuses on some of these undervalued aspects, especially in the light of novel ideas concerning the identity of a putative membrane receptor of $\mathrm{JH}$ and the mode of action of $\mathrm{JH}$ at the membrane level of the cell, in particular in the context of $\mathrm{Ca}^{2+}$-homeostasis and inhibition of apoptosis [16].

\section{Historical background of the discovery of the PTTHs}

\section{The bioassay used}

To test the biological activity present in the different chromatographic fractions obtained during the purification of the prothoracicotropic hormone (PTTH) of the silk moth B. mori, the assays were not done in Bombyx itself but in the saturniid moth $S$. cynthia ricini. Upon surgical removal of brains shortly after pupation, these moths remained as pupae for a long time without undergoing adult development [31]. Transplantation of live Bombyx brains and injection of Bombyx brain extracts into debrained Samia pupae caused the resumption of adult development, in exactly the same way as brainless Bombyx pupae responded. The reason for using this heterologous bioassay was a practical one; it was easier and Hironori Ishizaki had extensive experience with PTTH research with this species.

Initially the fact that Bombyx brain material was also active in Samia resulted in the assumption that PTTH was not speciesspecific [32]. However, it soon became clear that this was not the case. The first active factor that was identified turned out to be bombyxin, a $5 \mathrm{kDa}$ protein. It comprised many molecular forms which could only be satisfactorily resolved later by high performance chromatography. It took 650,000 Bombyx heads to finally obtain $50 \mu \mathrm{g}$ of pure bombyxin-I, the most abundant of all bombyxin isoforms. Later work showed that bombyxin belongs to the insulin superfamily and that it comprises many molecular forms coded for by a few dozen genes. In vitro, bombyxin-I proved to enhance ecdysone release from prothoracic glands dissected from freshly ecdysed Samia pupae at a threshold concentration of $10^{-11} \mathrm{M}$.

The most surprising result however was that bombyxin was totally inactive when tested in Bombyx pupae instead of in Samia. Further analysis of the crude extract revealed that it contained two distinct molecules: the $5 \mathrm{kDa}$ fraction (=bombyxin), only active in Samia, and a much larger molecule of approximately $30 \mathrm{kDa}$, that is active in Bombyx and other arthropods. The latter is referred to as big PTTH or the true PTTH, although its distribution in insects and arthropods is not at all ubiquitous [45]. Additional small PTTHs have since been discovered [45]. The chemical identity of an autocrine factor that activates ecdysteroidogenesis in the PGs of Bombyx [23] and Schistocerca [67] remains to be determined.

Extensive studies in Manduca sexta, mostly performed by the team originally headed by Larry Gilbert, largely confirmed the data that were first obtained in Bombyx [57]. These results now underlie the paradigm that the PGs are the major site of ecdysteroid synthesis and that this synthesis is mainly regulated by the brain hormone PTTH, in particular with respect to controlling the biosynthesis of ecdysteroids that play a role in metamorphosis.

\section{Chromatography used for purifying PTTH, bombyxin and other} ecdysiotropins

Both PTTH and bombyxin of Bombyx were purified by size exclusion chromatography using e.g. various types of Sephadex [31]. If small peptides exhibiting biological PTTH-activity would have been present, they would have ended up in the void volume, probably unnoticed. Later, for the further analysis of all the variants of bombyxin, HPLC techniques have been used.

Because nearly all known peptide hormones of insects are small in size ( 3 to a few dozen amino acids in length) Vandersmissen [66] hypothesized that, perhaps, some insects may have PTTH-active peptides that are even smaller than bombyxins. In this study, the desert locust Schistocerca gregaria served as experimental model (Fig. 1).

Locusts are interesting animals for the study of the physiology of the PGs because of the occurrence of phase polyphenism which means that in a given species the morphology, physiology and behavior can drastically change under the influence of the environment. Locusts can live solitariously as loners, or gregariously, in swarming groups. It has long been known that there are differences in morphology and persistence of PGs in the adult stage between the two phases (Fig. 1C-F). The JH titer in solitarious animals is known to be higher than in gregarious ones.

When Vandersmissen [66] investigated in which tissues of last nymphal instar $S$. gregaria ecdysteroidogenesis could be stimulated by brain extracts, he found that several tissues can produce small amounts of ecdysteroids, but that two tissues are potent producers, the PGs as expected, but also the gonads of both sexes. Using standard extraction (acidic methanol) and HPLC-identification methods, he demonstrated the presence of a small factor that potently stimulated ecdysteroid release from both the PGs and the gonads.

This factor is not only present in the brain, but also in the corpora cardiaca and the suboesophageal ganglion. It is definitely not a protein. The initial mass spectrometric analyses suggested that the active factor might be a peptide belonging to the AKH family. AKHs are very abundant in locusts, making them contaminants in several chromatographic fractions. However, upon testing many possible candidate peptides, none turned out to be active $[20,66]$. Therefore, the small locust ecdysiotropic gonadotropin remains to be identified.

\section{Less well documented functions of the PGs}

\section{PGs: to some extent the counterpart of the vertebrate thymus?}

Is disintegration of the PGs after metamorphosis beneficial for insects? To answer this question, one should know which molecules are released from the PGs. Ecdysteroid production, in particular at the end of the life of the PGs, is probably only part of their synthetic activity. In general, part of the biosynthetic pathway of steroids requires the presence of smooth endoplasmic reticulum (SER), the organelle where the synthesis and/or transformation of cholesterol and some enzymatic activities (e.g. in lipid synthesis) take place. This is known from both ultrastructural and biochemical studies [2]. In locusts, this is not so clear cut. In fact PGs of locusts do have a well-developed rough endoplasmic reticulum (RER), indicative of protein synthesis for release, while the SER is all in all only poorly developed (Fig. 3). A proteomic and peptidomic analysis of the PGs of the desert locust S. gregaria showed that these glands do not release any specific peptide [4]. This proteomics analysis was performed before any locust full genome had been sequenced, and made use of an EST-database. The results showed that, in addition to typical protein synthesis-related proteins, a number of proteins with a role in detoxification processes were present. This suggests 

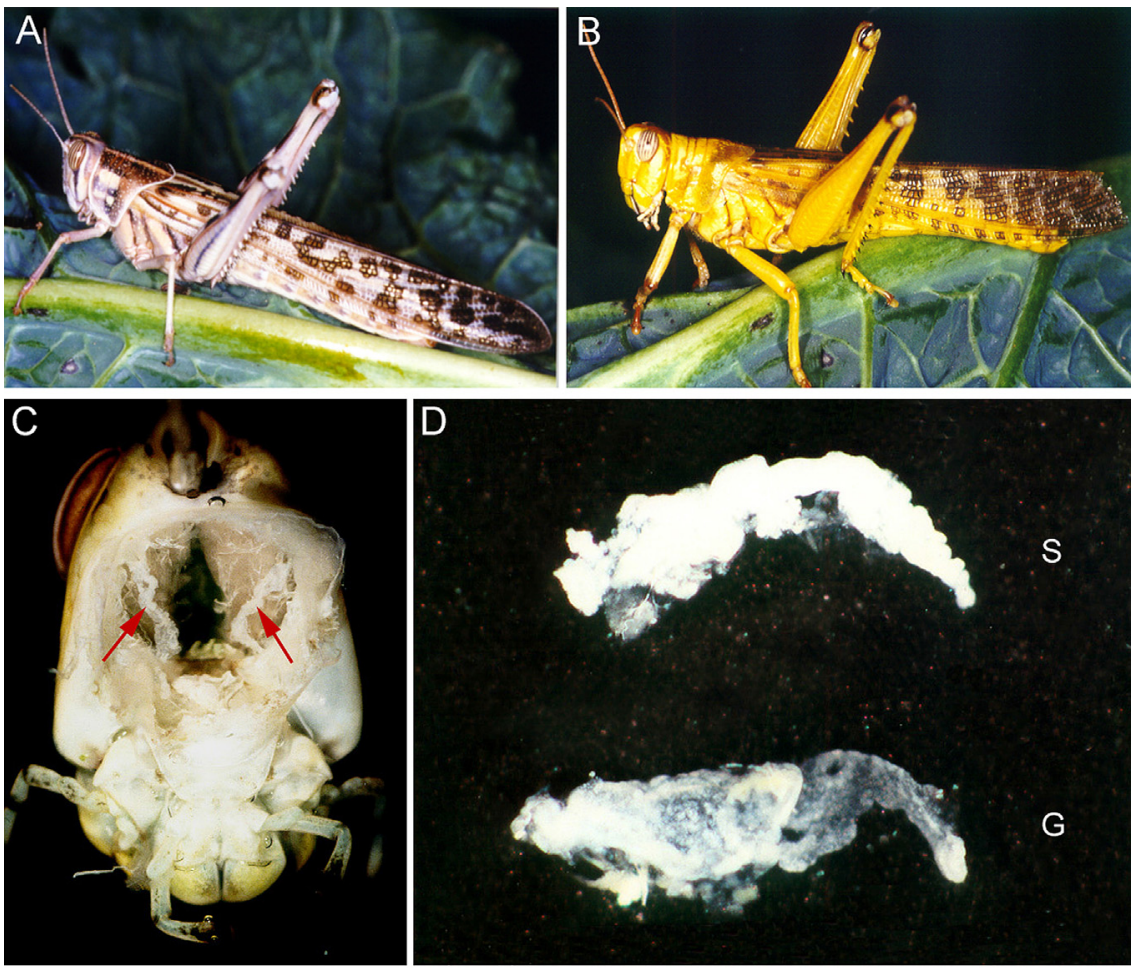

E

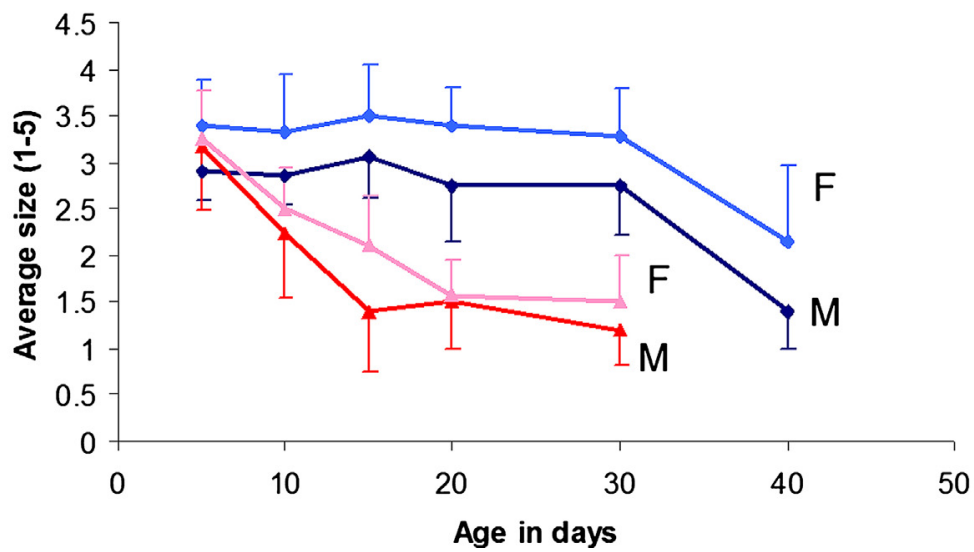

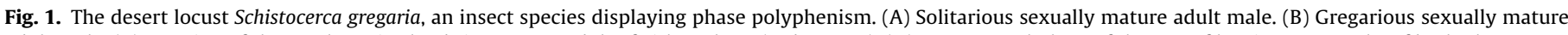

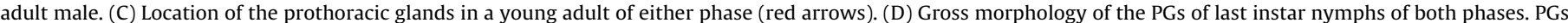

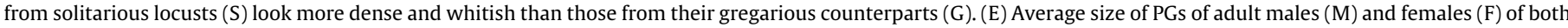

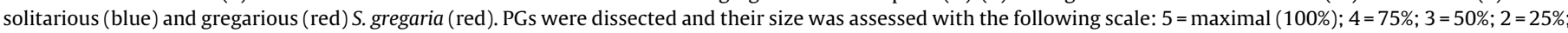
$1=$ residual; 0 = absent.

Unpublished data of M. Breuer cited, with permission, in Ref. [66].

some role for the PGs in the defense system. These data made Boerjan et al. [4] hypothesize that before the PGs degenerate, they may, to some extent, act as the functional counterpart of the thymus of jawed vertebrates (Gnathostomata), in particular of mammals. In juveniles the thymus plays a role in building up the immune system. Indeed, the thymus is the production site of T-cells which direct many aspects of the adaptive immune system. The production of thymocytes stops around puberty. From then on, the thymus degenerates in most species. The hormonal function of the thymus by means of (some of the different types of) thymosin is the only one that persists in elderly individuals [70]. Very recently it has been found that lowered activity of a single transcription factor, namely of thymic epithelial cell-specific Forkhead box N1 (FOXN1), is responsible for this decline in the functioning of the thymus in aging mice. This was shown by experiments in which forced upregulation of FOXN1 in the fully involuted thymus of aged mice resulted in robust thymus regeneration [6]. In insects, transcription factors belonging to the forkhead family occur, but to our knowledge no data with respect to the PGs have as yet been reported in the literature.

PGs may well have a function other than massive ecdysteroidogenesis at the end of larval life. We think that this massive ecdysteroidogenesis is in fact - in anthropomorphic wording - 'a death rattle' of which the functional importance has been overestimated in the past. We agree with the view of Mané-Padróz et al. [43] that the appearance of ecdysteroid peaks in the hemolymph signals that somewhere in the body relatively massive apoptosis is taking place. The number of ecdysteroid peaks present during metamorphosis may give an indication about the timing of apoptosis in different tissues. Briers et al. [8] measured ecdysteroid concentrations 
during metamorphosis of four fly species. In one species, one large peak occurs. In others there are up to three distinct peaks. Yet all four species metamorphose in a very similar way.

In most but not all mammalian species, the thymus gland that plays a key role in the development of the immune system in juveniles starts atrophying by involution when the individuals reach sexual maturity. Involution, the process by which functional tissue is replaced by fat, is thought to be one of the causes of aging. High levels of sex steroids induce this involution [39,75]. It should be noted that in insects ecdysteroids not only function as molting hormone but as sex steroids as well [12]. Perhaps, the developmental timing of the onset of disintegration from puberty on, i.e. when sex steroid hormone concentrations increase, of both the thymus in vertebrates and of the PGs in insects may be based upon the very same evolutionarily old signaling system, namely $\mathrm{Ca}^{2+}$-homeostasis with a special role for endogenous farnesol-like sesquiterpenoids This system has been outlined by [16]. For vertebrate physiologists farnesol may be a noble stranger although it is omnipresent in all cells of the vertebrate body as a precursor in the biosynthetic pathway of cholesterol. Up to 2014 when De Loof et al. [16] rediscovered that the $\mathrm{Ca}^{2+}$-ATPase (SERCA pump) blocker thapsigargin is, like farnesol and $\mathrm{JH}$, a sesquiterpenoid, it had been completely overlooked that farnesol might have a role by itself. According to [16], farnesol likely acts as a sort of gatekeeper to $\mathrm{Ca}^{2+}$-ATPase, ensuring free access of ATP to the enzymatically active site of at least some isoforms of $\mathrm{Ca}^{2+}$-ATPase. Absence of $\mathrm{JH}$ mimics the apoptosis-inducing effect of thapsigargin. Most probably the binding site on the $\mathrm{Ca}^{2+}$-pump for farnesol-like compounds is not specific but promiscuous, meaning that other lipophilic signaling molecules may compete with farnesol/JH. This view is supported by the fact that about 4200 synthetic JH analogs elicit similar effects as endogenous farnesol and $\mathrm{JH}$ [58]. Another argument is that some synthetic gonado-inhibiting benzodioxoles, when applied along with JH in the Galleria bioassay for detecting JH activity, can overrule the effect of $\mathrm{JH}$. This suggests that they interfere with the same receptor [65]. Furthermore, $\mathrm{Ca}^{2+}$-ATPases have 10 transmembrane domains. Perhaps specific sequences in some of these domains act as receptors for some lipophilic ligands. Sex steroids are probably physiologically important candidate players in controlling $\mathrm{Ca}^{2+}$ homeostasis [62]. If sex steroid competition results in less efficient removal of excess $\mathrm{Ca}^{2+}$, which is very toxic to any cell type, the risk of damage to macromolecules (a major cause of aging) as well the probability of entering the $\mathrm{Ca}^{2+}$-induced apoptosis cascade [49] will increase.

\section{PG- and thymus disintegration: advantageous or deleterious?}

Hitherto the question concerning the functional advantage, if any, of thymus- and PG disintegration remains largely unanswered. Maybe such disintegration is not beneficial at all, but a side effect of another developmental process with non-lethal effects. In adult solitarious locusts the PGs persist longer and look in better health than in gregarious ones (Fig. 1). However neither the solitarious nor the gregarious PGs produce ecdysteroids (see below). Apparently the higher JH titer which is typical for solitarious animals is causal to this phenomenon (see later). Solitarious animals live longer and are more fecund than gregarious ones. Thus, the persistence of PGs does not result in any damage. In some vertebrate species, the thymus persists as well in adult life, apparently without any clear negative effects. According to [6] a persistent functional thymus might be beneficial.

\section{PTTH in adults}

For a long time, the possibility that PTTH might continue to be present in adults was not even considered. Indeed, once the PGs have degenerated, why should PTTH production persist? Rybczynski et al. [57] reported that Manduca brains contain PTTH at all stages examined post-PG apoptosis, i.e. pharate adult and adult life, and that reproductive and reproduction-associated organs might be the targets of PTTH. It now gradually becomes clear what the function of PTTH might be in adults, namely a role in reproduction.

\section{PTTH and reproduction}

Ecdysteroid production not only continues throughout adult life of insects, but of other arthropods as well, e.g. of Crustacea, Myriapoda and Chelicerata. Apterygote insects continue molting after each reproductive cycle, illustrating that already long ago in evolution, ecdysteroids played a role in both reproduction and molting. The synthesis of ecdysone (E) in gonads, in particular in ovaries, is well documented. Several gonadotropins have been identified. They are all relatively small peptides. Hitherto PTTH has not been advanced as a candidate gonadotropin in females. The reason may be that the extraction methods that were used destroyed big proteins like PTTH. To better understand the possible link between PTTH and reproduction, some insight into the evolution of sexual reproduction may be helpful.

Some introductory endocrinological- and physiological archeology. At first sight, there is no relation whatsoever between the two major target sites of PTTH, the PGs and the reproductive system (see below), except that they are major producers of ecdysteroids. Yet, from an evolutionary point of view, there may be a link through the defense/immune system. Steroids in general are stress hormones. Glucocorticoids, in particular cortisol, are the best documented stress hormones of vertebrates; reproductive steroids are mild stress hormones [18]. Ecdysteroids can be considered as stress hormones because of their role in molting. Reproduction is usually not seen as a stress condition, to the contrary. However, if one analyzes reproduction from the viewpoint of physiology instead of that of evolutionary fitness, one inevitably will reach the conclusion that reproduction cannot be understood without invoking several key immunological principles [18]. Over a century ago, August Weismann (1834-1914) advanced the bright but counterintuitive idea that the body (somatoplasm) fights its own germ plasm, without knowing which mechanisms were responsible for this hostile behavior. To date, they are known. Sexual reproduction likely results from an ancient bacterial infection. The probable culprit was the premitochondrion, as shown by classical research on the formation of the primordial germ cells (PGCs) in Drosophila. Mitochondrial secretion products contribute to prevent the PGCs from integrating into an epithelium $[3,37,38]$, the basic tissue type of all animals [10]. In addition, PGCs behave as if they express in their plasma membrane an epitope(s) that makes them look like giant foreign (microbial) invaders. This combination elicits a series of evolutionarily ancient immune reactions: encapsulation of the PGCs by follicle cells, sex steroid production by follicle cells that eventually undergo apoptosis, yolk proteins with their agglutinin/lectin properties and even fertilization. On the other hand, immune reactions may have given rise to 'carrier proteins' to protect and carry steroid and sesquiterpenoid molecules from attack. Thus, PGs and gonadal follicle cells (in particular in ovaries) have in common that they can be hormonally forced to enter the apoptotic pathway. In PGs, the role of the hormone PTTH herein is established. In ovaries more research is needed to determine whether PTTH also plays a role.

PTTH: an evolutionarily ancient gonadotropin? The link gonadotropin, apoptosis, steroidogenesis. In locusts, the gonads start developing in the nymphal stadia. As cited before, Vandersmissen [66] demonstrated that not only the PGs of $S$. gregaria but the immature gonads 


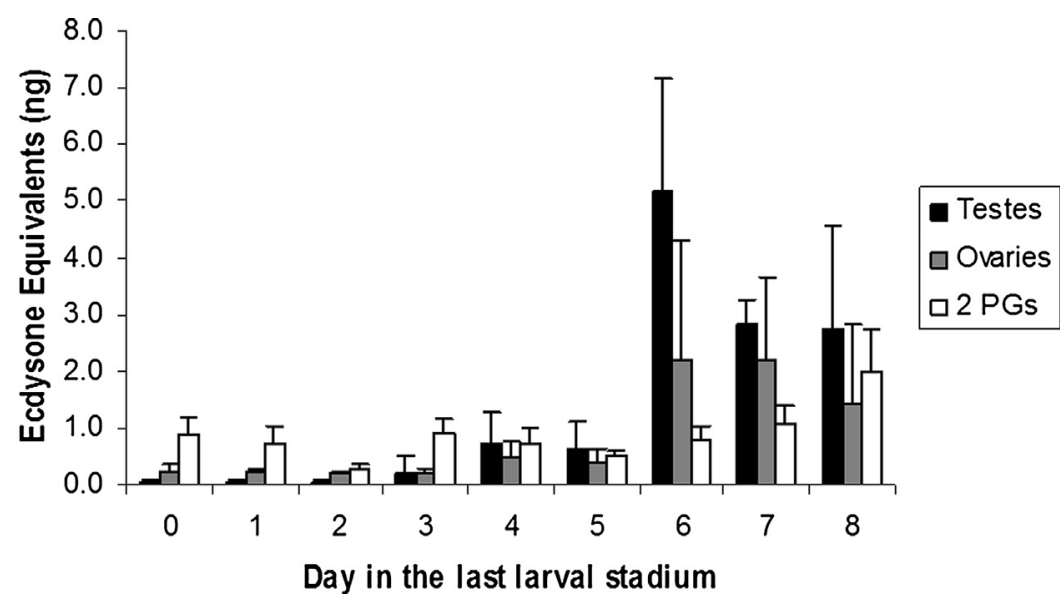

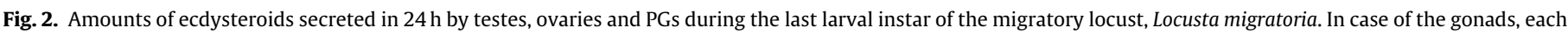
data point represents mean values \pm SD of 10-12 individual measurements, the data for the PGs are the results of 5 separate measurements. From [66].

of both sexes as well are potent producers/releasers of ecdysteroids (Fig. 2). Hence, it can be concluded that an ecdysiotropic gonadotropin is already at work in immature stages of this species.

Hentze et al. [29] observed that in adult Tribolium the genes required for the biosynthesis of ecdysone (E) are expressed in the tubular accessory gland cells of adult males. In contrast shade, the Halloween gene encoding the enzyme that converts E into 20hydroxyecdysone (20E) is not expressed in male accessory glands but only in females, in the ovaries. This contrasts with the data of [52] that showed that in the mosquito A. gambiae the male accessory glands but not the testes produce high amounts of $20 \mathrm{E}$ and deliver it to females during mating. It is even more intriguing that it has been reported that in the very same insect species the male accessory glands are also a site of synthesis of JH III and I [5]. De Loof et al. [17] hypothesized that $E$ is the counterpart of testosterone and $20 \mathrm{E}$ the counterpart of estrogens of vertebrates. A clear argument for the latter is that in several fly species administration (injection or oral uptake) of $20 \mathrm{E}$ to males induces vitellogenin synthesis, like estrogen administration does in male birds. This was first described in the fleshfly Sarcophaga (=Neobellieria) bullata [30]. Stoppie et al. [61] reported that feeding 20E to male Sarcophaga induced a rather extensive RER in the fat body while JH was completely inactive in this respect. Hentze et al. [29] also found that Torso is specifically expressed in the male accessory glands of adult Drosophila. Torso is a receptor tyrosine kinase. Its first detected role was in regulation of embryonic terminal cell fate in Drosophila [54]. It acts as the receptor for PTTH (see below). These findings unite the role of ecdysteroids in immature stages and in adults: the common denominator is that PTTH induces E synthesis in (all?) tissues that express Torso.

It is generally assumed that peak concentrations of steroid hormone which occur in blood in certain developmental stages are necessary for bringing about a particular function. For example, the high ecdysteroid peak(s) in last larval stadia insects are thought to be necessary for realizing metamorphosis. When the methods for silencing genes in the biosynthetic pathway of $\mathrm{E}$ and $20 \mathrm{E}$ became available, the question of the supposed necessity for such peak concentrations could be addressed. When Marchal et al. [44] silenced some of the Halloween genes in S. gregaria, the normal ecdysteroid peak at the end of the instar was abolished, but molting to the next instars' was still normal. This means that one should be careful in attributing a role to a particular concentration of a steroid hormone. Local high concentrations of e.g. JH and or E in male accessory glands may have a different role, e.g. in epigenetics $[9,14]$, compared to low concentrations elsewhere in the body.

\section{PTTH: Reproduction versus diapause}

In the cotton bollworm, Helicoverpa armigera, as in many other insect species, developing into adults or entering diapause is controlled by day length. Short day length is usually causal to diapause induction. Recombinant PTTH was effective in breaking pupal diapause [69]. By various experiments Lu et al. [42] have shown how changes in day length via specific transcription factors affect the expression of the PTTH gene, thereby regulating developmental
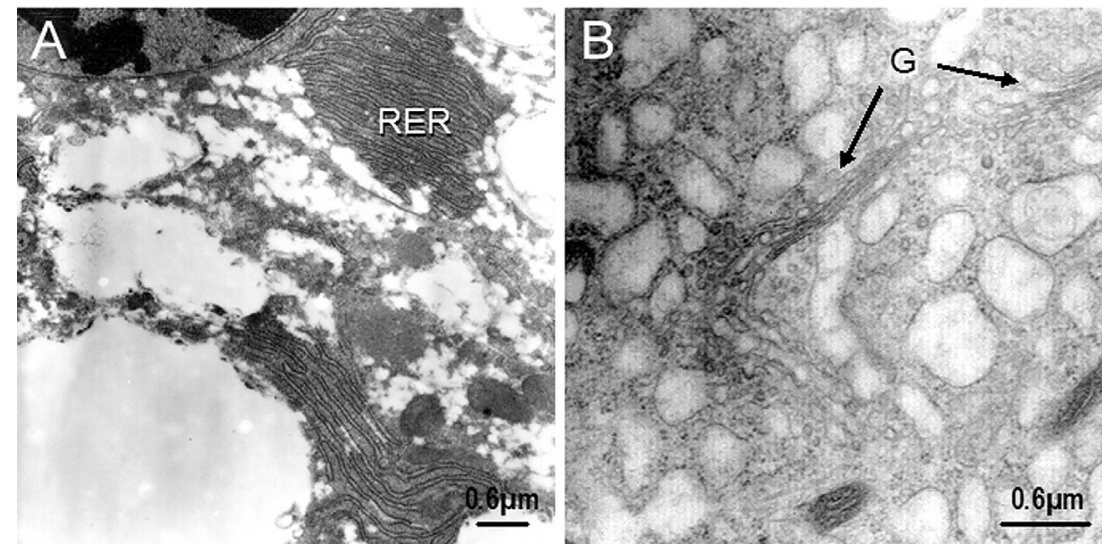

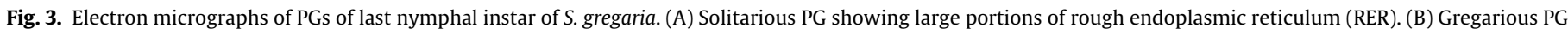
showing Golgi apparatus (arrow). This shows that in both phases the PGs synthesize and secrete proteins. The identity of these proteins is not known. 
timing (i.e. induction of diapause). In the corn earworm Helicoverpa zea, both PTTH and diapause hormone (DH) are abundant from pupation until adult eclosion, while in pupae that enter diapause PTTH transcripts are undetectable. Injection of either ecdysone or of DH can terminate pupal diapause in this and related species [74].

In the cabbage army moth Mamestra brassicae, the hemolymph PTTH titer is maintained at high levels after pupation in nondiapause pupae, while the titer in diapause pupae drops to an undetectable level. The PTTH content of the brain in post-pupation brain was higher in diapause animals than in non-diapause animals. This means that diapause-destined animals can produce PTTH but that they do not release it into the hemolymph. A lack of PTTH is necessary and sufficient for inducing pupal diapause in this species [46].

In the northern house mosquito, Culex pipiens, on the other hand, PTTH cycles during the day in both long day (non diapausedestined) and short day (diapause-destined) last instar larvae. This cycling disappears in pupae, but PTTH transcripts were slightly higher in short-day pupae than in long-day pupae throughout much of the pupal stage. PTTH transcripts were low in both nondiapausing females and postdiapause females, on condition they were denied a blood meal. Upon ingesting a blood meal, the prerequisite condition for ovarian development, PTTH transcripts rose approximately 7 -fold in $2 \mathrm{~h}$, and remained elevated for $24 \mathrm{~h}$. In force-fed diapausing females there was also a substantial increase. It would be interesting to investigate in which tissue(s) the PTTH receptor is expressed in blood-fed adults [73].

When young adults of the Colorado potato beetle, Leptinotarsa decemlineata, were neck-ligated, the ecdysteroid titer in the hemolymph rose by about $500 \%$ in about one day [7]. It is the absence of $\mathrm{JH}$ that causes this increase. This follows from the fact that beetles raised under short day conditions prepare for entering adult diapause and have much higher ecdysteroid titers than nondiapause destined reproducing adults. Because adult beetles do not molt anymore, the high ecdysteroid titers in diapausing animals must have a function other than inducing molting. Many changes occur when preparing for diapause. For example, compared to its counterpart in animals raised in long day conditions, in reproducing animals the structure of the fat body drastically changes. Lipids accumulate and large protein granules are also deposited, apparently by a deficiency in the final steps of the protein secreting system due to absence of JH [19]. Diapause can last for over a year without affecting the total longevity of the beetles. High ecdysteroid titers during diapause or during long-lasting metamorphosis may have an as yet unknown role in retarding aging and keeping metabolism at a low level. No data are available on the presence of a PTTH-like hormone in Leptinotarsa.

Release of both bombyxin and PTTH from the brain has a strong circadian rhythm driven by master clock cells in the brain of Rhodnius prolixus [64]. By regulating the expression of the PERIOD protein, these rhythms synchronize the photosensitive brain clock with the photosensitive peripheral clock in the cells of the PGs in which both clocks regulate steroidogenesis. In peripheral tissues of Rhodnius such as the salivary glands and the fat body, PTTH and bombyxin alone do not influence PERIOD but in combination they do, implying that their signaling pathways converge [64]. In the moth Antheraea pernyi, the clock controlled gene nat that codes for $\mathrm{N}$-acetyltransferase has been shown to be causally linked to the release of PTTH, resulting in termination of diapause [48].

Another aspect of the relationship between light and PTTH has been highlighted by [71]. They showed that in Drosophila PTTH is causal to light avoidance at the end of larval life. This enables larvae to move to a darker environment to initiate the immobile maturation phase. Like ecdysteroidogenesis, this effect is also mediated by the Torso receptor.

\section{PGs, PTTH, CA and JH}

\section{PTTH and bombyxin in nerve fibers in or around the $C A$}

In the brain of $B$. mori, four pairs of large dorsomedial neurosecretory cells contain immunoreactive bombyxin. The reactive material could be traced down through the axons that emerge from these neurosecretory cells and cross at the central line of the brain so that the axons reach the contralateral corpus allatum. The axon endings were localized in the periphery of the CA. This localization suggests that bombyxin is released, according to [47] probably in a pulsatile way, from the CA into the hemolymph [32].

A monoclonal PTTH antibody immunostained two pairs of dorsolateral neurosecretory cells in the Bombyx brain. It also stained axons from these cells that run across the brain midline to reach the contralateral CA. In contrast to the situation with bombyxincontaining neurons which are preferentially localized in the surface region of the CA, the PTTH axon terminals penetrate the CA. This means that in Bombyx and in Samia, where a similar immunolocalization has been observed, the CA serve as the neurohemal organ for PTTH release as well [32]. Agui et al. [1] have shown that in $M$. sexta, the CA is also the site where PTTH-active material is released into the hemolymph.

This raises an intriguing question: do at some time in larval development PTTH and bombyxin have an effect - stimulating or inhibitory - on the release of JH or any other (putative) functional molecule from the CA as well? Ecdysteroids are known to have an inhibitory effect on JH production [60]. Or could it be that electrical activity in the axons somehow influences CA activity? We did not find any report on this topic in the literature. There may exist a complex feedback loop involving PTTH, ecdysteroids and JH.

\section{Application of synthetic JH inhibits ecdysteroidogenesis in PGs and metamorphosis}

It is known since the early days of research on the CA that in most species allatectomy in an early larval instar precociously induces metamorphosis. Implantation of extra CA and application of synthetic $\mathrm{JH}$ or JH-analogs (JHA) usually extend larval life. In some species $\mathrm{JH}(\mathrm{A})$ can induce supernumerary larval instars [59]. All this indicates that high $\mathrm{JH}$ titers inhibit the PTTH-PG axis and metamorphosis. One possibility is that high JH titers inhibit the release of PTTH, but this is likely not the case, as was demonstrated by Gu et al. [24] in B. mori. Another possibility is that JH acts on the PGs themselves. PGs in JH-1 or hydroprene (JHA)-treated larvae showed no response in ecdysteroidogenesis to PTTH. In contrast, allatectomy on day 0 of the last larval instar accelerated development. Within one day of allatectomy, the PGs had become responsive to PTTH. The authors concluded that absence of JH is a prerequisite for successful PTTH signal transduction. Maybe the explanation for the refractoriness of PGs to PTTH in certain developmental stages [41] can in part be due to an inhibitory effect of JH on PTTH-activation of ERK. Gu and Tsai [26] also reported that decreased JH biosynthesis is related to precocious metamorphosis in a mutant of Bombyx.

As cited before, neck ligation in adult $L$. decemlineata causes a very fast and drastic rise in ecdysteroid titer. This experiment clearly showed that no brain hormone is required for stimulating ecdysteroid biosynthesis and release; absence of $\mathrm{JH}$ is essential and sufficient.

In the moth Sesamia nonagrioides, several larval molts can occur without a brain. The fact that larval molts can occur in the absence of the CA suggests considerable JH persistence, apparently due to very slow degradation by JH-esterases. Furthermore, an unknown head factor outside the brain is needed for the pupal-adult molt $[50]$. 
Again in Sesamia, the gene encoding PTTH is expressed in the brain and, remarkably also in the gut. Larvae and pupae can molt without a brain but the PGs must be present. Decapitation increases the expression of PTTH in the gut. According to [51] molts of this species are driven by PTTH from the gut. In our view, absence of JH could as well be the driving force. The alimentary canal is a place where programmed cell death is part of the normal physiology. Perhaps, enabling apoptosis is the key function for gut-PTTH.

\section{Ecdysteroidogenesis and $\mathrm{Ca}^{2+}$-homeostasis}

The involvement of $\mathrm{Ca}^{2+}$ in the PTTH-signaling cascade is well documented in both $M$. sexta [21,56] and B. mori $[25,27,28]$. De Loof et al. [16] hypothesized that the long sought for membrane receptor of $\mathrm{JH}$ could be attributable to (some isoforms) of $\mathrm{Ca}^{2+}$-ATPase. If correct, this assigns a key role to $\mathrm{JH}$ in $\mathrm{Ca}^{2+}$-homeostasis, namely in keeping the cytosolic $\mathrm{Ca}^{2+}$-concentration low by stimulating $\mathrm{Ca}^{2+}-$ ATPases to pump excess $\mathrm{Ca}^{2+}$ out of the cells. PTTH signaling also involves $\mathrm{Ca}^{2+}$, but in a different way. Indeed, it involves the influx of $\mathrm{Ca}^{2+}$. Evidently other downstream effectors in the signaling cascade are needed. The conceptual difficulty is that it is not the presence, but the absence of $\mathrm{JH}$ that is causal to initiation of metamorphosis. This raises the question of how the absence of a hormone can transduce a signal. Because hormones need a receptor to transfer their message, which type of receptor can deliver a message when its ligand is absent?

The model we will propose requires insight into the basic principles of development, of the nature of the PTTH receptor and of some basic principles of $\mathrm{Ca}^{2+}$-homeostasis.

\section{Development: generating differences in plasma membrane properties}

Tissue-specificity of peptide/protein hormone action is primarily based upon the fact that all cells of the body have the same genome (a few exceptions not taken into account) but differ in their plasma membrane-cytoskeletal properties. Its essence is: Differentiation: Keep the genome constant but change its ionic and/or macromolecular environment over and over again [11]. The successive steps in this process of differentiation, in particular the double asymmetry principle as well as the key role of the plasma membrane in control of gene expression have been outlined by $[11,15]$. The double asymmetry principle says that first the egg is made non-spherically symmetrical, mainly by rearrangements of components of its cortical layer. This is clearly visible in eggs in which this cortical layer contains pigment granules, e.g. in frog eggs. The second asymmetry element is that the egg very soon, not later than the third cleavage round, starts cleaving asymmetrically. The combination of both asymmetry generating principles makes that already from very early in development cells are formed which differ in their membrane properties. For figures illustrating this principle, see [15]. This membrane-asymmetry gets more and more pronounced as development proceeds. Hence, it is absolutely normal that only certain cell types express a particular receptor, e.g. PGs and gonads expressing Torso.

\section{Torso (Tor): the membrane receptor for PTTH}

The membrane receptor for PTTH is Torso (Tor). Tor has structural similarities to growth-factor tyrosine kinases except that the extracellular domain does not resemble that of other known receptor tyrosine kinases [40]. Tor has two major functions.

First, Tor plays a key role in patterning of the anterior and posterior axis (termini) of the early embryo. Tor is uniformly distributed throughout the embryo, indicating its activation must be achieved locally at the termini. Previously Trunk, which is structurally related to PTTH probably due to a duplication of an ancestral gene [22,54], was thought to be the functional ligand. Later, Torso-like was advanced as the most probable ligand [35]. This protein is the sole Drosophila member of the membrane attack complex/perforin protein superfamily, generally known for pore-forming and immune defense.

Second, later in development Tor is specifically expressed in the PGs of larvae. Here, its ligand is no longer Torso-like but PTTH [54]. In PGs of the silkworm B. mori, Tor-mRNA levels were high in the beginning and also at the end of the last larval instar. They were low in both the mid instar and the pupa. In the in vivo situation, the decline in PTTH mRNA level roughly parallels the one of JH up to day 8 of the last larval instar. Later, the JH titer remains very low while the level of Torso mRNA rises sharply until day 10, and falls dramatically in the pupa. The ecdysteroidogenic activity in the PGs also rises sharply from day 8 on to reach high values in the pupa.

Adding PTTH to PGs of day-6 last instar larvae in vitro, was shown to induce a significant decrease in Tor mRNA levels $1 \mathrm{~h}$ after the start of the treatment. It lasted for another hour. When the JHA methoprene was applied to newly ecdysed last instar larvae, a decline in Tor mRNA levels during the early last larval instar was delayed compared to the acetone controls. Injection of 20E increased Tor mRNA levels. These levels were also nutritionsensitive. Thus, Tor mRNA levels may play a role in regulating ecdysteroidogenesis by PGs during development [72].

\section{The role of $\mathrm{JH}$ in $\mathrm{Ca}^{2+}$-homeostasis}

Gu et al. [24] demonstrated that in B. mori, JH inhibits ecdysteroid release by PGs. Gu et al. [25] also showed that in Bombyx ERK phosphorylation by PTTH was partially reduced in $\mathrm{Ca}^{2+}$-free medium and that calmodulin plays a role. Furthermore when PGs were treated with agents that directly elevate $\left[\mathrm{Ca}^{2+}\right]_{i}$ (e.g. thapsigargin or A23187), a great increase in ERK phosphorylation was observed. We think that the essence of the mode of action of PTTH and JH on both ecdysteroidogenesis and apoptosis resides in the principles of $\mathrm{Ca}^{2+}$ homeostasis [16]. One should always keep in mind that $\mathrm{Ca}^{2+}$ is very toxic. Cells must therefore constantly pump $\mathrm{Ca}^{2+}$ out of the cytoplasm; otherwise $\mathrm{Ca}^{2+}$-induced apoptosis will result [49]. If, by whatever mechanism, $\mathrm{Ca}^{2+}$ flows into a cell, two major responses are possible: (1) local increase in $\left[\mathrm{Ca}^{2+}\right]$ causes local changes in configuration and activity of some macromolecules, and by doing so, a rise in $\left[\mathrm{Ca}^{2+}\right]_{i}$ acts as a secondary messenger system. This effect must be short-lived, otherwise cellular damage will occur. $\mathrm{Ca}^{2+}$-ATPases will be activated by the influx and pump the excess $\mathrm{Ca}^{2+}$ away. (2) In case the $\mathrm{Ca}^{2+}$-influx is substantial and lasts long so that the $\mathrm{Ca}^{2+}$-ATPases present in the plasma membrane cannot counter the influx, $\mathrm{Ca}^{2+}$-induced $\mathrm{Ca}^{2+}$ release from internal stores will be induced. Such local increases are propagated as waves throughout the cell $[33,34]$. If they last too long, $\mathrm{Ca}^{2+}$-induced apoptosis will result. In our opinion, this is what happens in the PGs of metamorphosing insects.

\section{The sequence of events in our model on the interplay between $\mathrm{PTTH}(\mathrm{s})$ and $\mathrm{JH}$ in ecdysteroidogenesis}

- The membrane protein Torso acts as the receptor for big PTTH. Upon binding, $\mathrm{Ca}^{2+}$ flows into the cell and activates its typical signaling cascade $[21,25,27,28,56]$. Not all developmental stages are equally susceptible to this activation.

- As long as the JH titer is high, $\mathrm{Ca}^{2+}$-ATPases can keep $\left[\mathrm{Ca}^{2+}\right]_{i}$ low, even if PTTH causes some $\mathrm{Ca}^{2+}$-influx in the PG-cells.

- At low cytosolic $\mathrm{Ca}^{2+}$ concentrations, part of the $\mathrm{Ca}^{2+}$ present in cells is confined to smooth endoplasmic reticulum membrane stores. Exactly this membrane system is usually well developed 
in steroid synthesizing cells. Apparently some enzymatic steps in steroid biosynthesis are inhibited at high intralumenal $\mathrm{Ca}^{2+}$ concentrations.

- When JH is cleared from the body in the last larval instar by esterases, $\mathrm{Ca}^{2+}$ is no longer removed as quickly as it enters the cells which expose the Torso membrane receptor. As a result $\left[\mathrm{Ca}^{2+}\right]_{i}$ starts rising and triggers $\mathrm{Ca}^{2+}$-induced $\mathrm{Ca}^{2+}$ release from the $\mathrm{Ca}^{2+}$-storage sites, a process that is very well documented in e.g. muscle contraction.

- One of the effects of this rise is that the inhibition of ecdysteroid biosynthesis and release which prevails as long as JH is present and $\left[\mathrm{Ca}^{2+}\right]_{i}$ is kept low is lifted.

- If the increase in cytosolic $\mathrm{Ca}^{2+}$ concentration lasts too long, $\mathrm{Ca}^{2+}$ induced-apoptosis will be initiated. High ecdysteroid concentrations are thought to have a role in bringing about programmed cell death/apoptosis in some tissues.

- PTTH in adults: in gonads apoptosis takes place in some cell types, e.g. in the follicle cells surrounding the ripening oocytes. Thus, even in gonads, a similar mechanism as in the PGs is likely at work. Apparently, an increased influx of $\mathrm{Ca}^{2+}$ by a gonadotropin is not counterbalanced in full by $\mathrm{Ca}^{2+}$-extrusion. The resulting higher $\left[\mathrm{Ca}^{2+}\right]_{i}$ is favorable for steroid production. It should be noted that the role of PTTH in gonads is not yet well experimentally documented.

\section{Discussion}

The first function that is discovered for a newly identified hormone will likely continue to direct research into its mode of action for quite some time. This happened with PTTH. Because in holometabolous insects the PGs usually degenerate after metamorphosis, and because ecdysteroid peaks were assumed to be necessary for molting and metamorphosis, the possibility that PTTH(s) might also occur in the adult stage was not even considered for a long time. As long as the relevant molecular biological tools were not available, it was also unclear whether or not PTTH occurs in all insect species. It does not [45]. Yet, also in insects that do not have PTTH or a close relative (e.g. Trunk), PGs do produce ecdysteroids and do disintegrate. In these species, the gonads use ecdysteroids as sex steroids as well $[12,13]$. It can therefore be expected that in these species other types of brain peptides exert similar effects as PTTH.

Intuitively one is tempted to think that disintegration of the PGs during or shortly after metamorphosis must have a function, otherwise it would not be so widespread. However, this may not be the case. In solitarious locusts, the PGs persist longer than in gregarious animals, without any visible negative effect. In the adult stage, neither solitarious nor gregarious PGs produce substantial amounts of ecdysteroids [63]. In our opinion, the massive ecdysteroid release by the PGs that precedes their disintegration in holometabolous insects in fact signals their imminent inactivation and death. Whether the disintegration of the PGs during metamorphosis has a chronobiological aspect remains to be demonstrated. Could it be that it helps to adapt the switch from a given photoperiod during larval life to another regime in the adult? Another hypothesis says that continuing ecdysteroid secretion from the PGs might perhaps interfere with the ecdysteroid production by the gonads themselves, for example by initiating vitellogenin synthesis too early in development? These and other remaining questions may be answered if one would succeed in finding a key transcription factor that is essential for continuing activity, as has been found in the thymus [6].

To date it is known that big PTTH is present in adults of some species in which it has been sought for. It likely plays a role in reproduction. The common denominator between effects of PTTH on PGs and gonads is that both express the Torso receptor gene. Thus, if one would nominate big PTTH as the activator of the Torso membrane receptor, one would let room for more than one function at the organ level.

The way of looking at reproduction as a strategy of the immune system [18] is undoubtedly counterintuitive, but it is the most logical one if reproduction is analyzed from the viewpoint of physiology. It is tempting to think that all processes in the body are maximally optimized. If the PGs resemble the vertebrate thymus and play a role in the defense system as suggested by [4], and if sex steroid production by the gonads can also be seen as an immune defense strategy, the fact that both PGs and gonads express Tor may make sense.

The presence of both PTTH and Torso in parts of the gonadal complex of adults of some species raises the question of the role of $\mathrm{JH}$. The general rule is that $\mathrm{JH}$ is required for gonad development and functioning in adults. This means that in adults the presence of $\mathrm{JH}$ inhibits ecdysteroidogenesis less than it does in juvenile stages. Apparently, in adults an as yet unknown mechanism permits more $\mathrm{Ca}^{2+}$ to enter the ecdysteroid producing cells than their ATPases can remove.

In our opinion, the role of absence of $\mathrm{JH}$ as being causal to PG disintegration has been largely overlooked in the past. This is due to the fact that the bioassays used for monitoring PTTH activity were performed in systems in which JH was absent, namely debrained pupae and PGs cultured in vitro. We advance absence of $\mathrm{JH}$ as the primordial trigger of metamorphosis and PTTH as a secondary actor, which reinforces the effect of absence of $\mathrm{JH}$ on $\mathrm{Ca}^{2+}$-homeostasis.

To date one can safely state that PTTH only triggers the Torso signaling cascade in PGs in instances in which the JH titer is low. During metamorphosis the JH titer in the hemolymph is very low. This does not exclude that in some places of the body, a very high concentration of JH can be found, e.g. in the male accessory glands of $H$. cecropia. This JH is transferred to the female during copulation. It may serve a role in epi-endocrinology [14] and in $\mathrm{Ca}^{2+}$-homeostasis of the ovarian follicles [16]. It is remarkable that male accessory glands of some species also turn out to be important sites of synthesis of ecdysone as shown by [29]. It strengthens the hypothesis that ecdysteroids are the evolutionarily ancient sex-steroids of insects, and probably of all Ecdysozoa.

\section{Conclusion}

The Torso receptor in the plasma membrane seems to act as a (warning) flag that signals which cells are susceptible to PTTH-Ca ${ }^{2+}$ induced apoptosis. PTTH release will initiate effects in the Torsoflag exposing tissues, but only if the JH titer drops to a very low value for a sufficiently long period of time, or if in the presence of $\mathrm{JH}$ (e.g. in adults) the influx of $\mathrm{Ca}^{2+}$ into the cytosol exceeds the amount of $\mathrm{Ca}^{2+}$ that can be pumped out of the cell by all $\mathrm{Ca}^{2+}$-ATPases. Cells that have a very low $\mathrm{Ca}^{2+}$ influx are probably not targets for PTTH. This model explains why PTTH can be present throughout life and why it can sort differential effects in a species-, tissue- (PGs, gonads, gut?) and developmental stage-specific way.

\section{Contributors}

Arnold De Loof and Elisabeth Marchal wrote the paper; Tim Vandersmissen provided the experimental locust data; Liliane Schoofs substantially contributed to the funding and coordination.

\section{Conflict of interest}

None of the authors has a conflict of interest. 


\section{Acknowledgements}

Thanks to the FWO Flanders and the Research Council of the KU Leuven (GOA) for sponsoring our locust research during many years.

\section{References}

[1] Agui N, Bollenbacher WE, Granger NA, Gilbert LI. Corpus allatum is release site for insect prothoracicotropic hormone. Nature 1980;285:669-70.

[2] Alberts B, Johnson A, Lewis J, Raff M, Roberts K, Walter P. Molecular biology of the cell. 4th ed. New York: Garland Science; 2002.

[3] Amikura R, Sato K, Kobayashi S. Role of mitochondrial ribosome-dependent translation in germline formation in Drosophila embryos. Mech Dev 2005;122:1087-93.

[4] Boerjan B, Vandingenen K, De Loof A, Schoofs L. In search for non-steroidogenic functions of the prothoracic glands of the desert locust, Schistocerca gregaria: a peptidomic and proteomic approach. Peptides 2012;34:57-64.

[5] Borovsky D, Carlson DA, Hancock RG, Rembold H, van Handel E. De novo biosynthesis of juvenile hormone III and I by the accessory glands of the male mosquito. Insect Biochem Mol Biol 1994;24:437-44.

[6] Bredenkamp N, Nowell CS, Blackburn CC. Regeneration of the aged thymus by a single transcription factor. Development 2014;141:1627-37.

[7] Briers T, Peferoen M, Deloof A. Ecdysteroids and adult diapause in the Colorado potato beetle, Leptinotarsa decemlineata. Physiol Entomol 1982;7:379-86.

[8] Briers T, Vanbeek E, Deloof A. Ecdysteroid activity during metamorphosis and in male and female adults of 4 blowfly species. Comp Biochem Phys A 1983;74:521-4

[9] Davey KG. The modes of action of juvenile hormones: some questions we ought to ask. Insect Biochem Mol Biol 2000;30:663-9.

[10] De Loof A. All animals develop from a blastula - consequences of an undervalued definition for thinking on development. Bioessays 1992;14:573-5.

[11] De Loof A. Differentiation - keep the genome constant but change over and over again its ionic and or macromolecular environment - a conceptual synthesis. Belg J Zool 1993;123:77-91.

[12] De Loof A. Ecdysteroids: the overlooked sex steroids of insects? Males: the black box. Insect Sci 2006;13:325-38.

[13] De Loof A. Ecdysteroids, juvenile hormone and insect neuropeptides: recent successes and remaining major challenges. Gen Comp Endocrinol 2008; 155:3-13.

[14] De Loof A, Boerjan B, Ernst UR, Schoofs L. The mode of action of juvenile hormone and ecdysone: towards an epi-endocrinological paradigm. Gen Comp Endocrinol 2013;188:35-45

[15] De Loof A, Callaerts P, Vanden Broeck J. The pivotal role of the plasma membrane-cytoskeletal complex and of epithelium formation in differentiation in animals. Comp Biochem Phys A 1992;101:639-51.

[16] De Loof A, De Haes W, Janssen T, Schoofs L. The essence of insect metamorphosis and aging: electrical rewiring of cells driven by the principles of juvenile hormone-dependent $\mathrm{Ca}^{2+}$-homeostasis. Gen Comp Endocrinol 2014; 199:70-85.

[17] De Loof A, Huybrechts J, Geens M, Vandersmissen T, Boerjan B, Schoofs L. Sexual differentiation in adult insects: male-specific cuticular yellowing in Schistocerca gregaria as a model for reevaluating some current (neuro)endocrine concepts. J Insect Physiol 2010;56:919-25.

[18] De Loof A, Huybrechts R, Kotanen S. Reproduction and love: strategies of the organism's cellular defense system. Comp Biochem Phys C 1998;120:167-76.

[19] De Loof A, Lagasse A. Juvenile hormone and ultrastructural properties of fat body of adult Colorado beetle, Leptinotarsa decemlineata Say. Z Zellforsch Mikrosk Anat 1970;106:439-50.

[20] De Loof A, Vandersmissen T, Huybrechts J, Landuyt B, Baggerman G, Clynen E, et al. APRP, the second peptide encoded by the adipokinetic hormone gene(s), is highly conserved in evolution. A role in control of ecdysteroidogenesis? Ann NY Acad Sci 2009;1163:376-8.

[21] Fellner SK, Rybczynski R, Gilbert LI. $\mathrm{Ca}^{2+}$ signaling in prothoracicotropic hormone-stimulated prothoracic gland cells of Manduca sexta: evidence for mobilization and entry mechanisms. Insect Biochem Mol Biol 2005·35:263-75.

[22] Grillo M, Furriols M, de Miguel C, Franch-Marro X, Casanova J. Conserved and divergent elements in Torso RTK activation in Drosophila development. Sci RepUK 2012;2:1-7

[23] Gu SH. Autocrine activation of ecdysteroidogenesis in the prothoracic glands of the silkworm, Bombyx mori. J Insect Physiol 2007;53:538-49.

[24] Gu SH, Chow YS, Yin CM. Involvement of juvenile hormone in regulation of prothoracicotropic hormone transduction during the early last larval instar of Bombyx mori. Mol Cell Endocrinol 1997;127:109-16.

[25] Gu SH, Lin JL, Lin PL. PTTH-stimulated ERK phosphorylation in prothoracic glands of the silkworm, Bombyx mori: role of $\mathrm{Ca}^{2+} /$ calmodulin and receptor tyrosine kinase. J Insect Physiol 2010:56:93-101.

[26] Gu SH, Tsai WH. Decreased JH biosynthesis is related to precocious metamorphosis in recessive trimolter (rt) mutants of the silkworm, Bombyx mori. Arch Insect Biochem Physiol 2012;79:235-46.

[27] Gu SH, Yeh WL, Young SC, Lin PL, Li S. TOR signaling is involved in PTTHstimulated ecdysteroidogenesis by prothoracic glands in the silkworm, Bombyx mori. Insect Biochem Mol Biol 2012;42:296-303.
[28] Gu SH, Young SC, Lin JL, Lin PL. Involvement of PI3K/Akt signaling in PTTHstimulated ecdysteroidogenesis by prothoracic glands of the silkworm, Bombyx mori. Insect Biochem Mol Biol 2011;41:197-202.

[29] Hentze JL, Moeller ME, Jorgensen AF, Bengtsson MS, Bordoy AM, Warren JT, et al. Accessory gland as a site for prothoracicotropic hormone controlled ecdysone synthesis in adult male insects. PloS ONE 2013;8:e55131.

[30] Huybrechts R, De Loof A. Induction of vitellogenin synthesis in male Sarcophaga bullata by ecdysterone. J Insect Physiol 1977;23:1359-62.

[31] Ishizaki H, Ichikawa M. Purification of brain hormone of silkworm Bombyx mori. Biol Bull 1967;133:355-68.

[32] Ishizaki H, Suzuki A. The brain secretory peptides that control moulting and metamorphosis of the silkmoth, Bombyx mori. Int J Dev Biol 1994;38:301-10.

[33] Jaffe LF. The path of calcium in cytosolic calcium oscillations - a unifying hypothesis. Proc Natl Acad Sci U S A 1991;88:9883-7.

[34] Jaffe LF. Classes and mechanisms of calcium waves. Cell Calcium $1993 ; 14: 736-45$.

[35] Johnson TK, Crossman T, Foote KA, Henstridge MA, Saligari MJ, Beadle LF, et al. Torso-like functions independently of Torso to regulate Drosophila growth and developmental timing. Proc Natl Acad Sci U S A 2013;110:14688-92.

[36] Karlson P. On the hormonal control of insect metamorphosis. A historical review. Int J Dev Biol 1996;40:93-6.

[37] Kobayashi S, Amikura R, Okada M. Presence of mitochondrial large ribosomal RNA outside mitochondria in germ plasm of Drosophila melanogaster. Science 1993;260:1521-4.

[38] Kobayashi S, Sato K, Hayashi Y. The role of mitochondrial rRNAs and nanos protein in germline formation in Drosophila embryos. Zoolog Sci 2005;22:943-54.

[39] Leposavic G, Perisic M. Age-associated remodeling of thymopoiesis: role for gonadal hormones and catecholamines. Neuroimmunomodulation $2008 ; 15: 290-322$

[40] Li WX. Functions and mechanisms of receptor tyrosine kinase torso signaling: lessons from Drosophila embryonic terminal development. Dev Dyn 2005:232:656-72.

[41] Lin JL, Gu SH. In vitro and in vivo stimulation of extracellular signal-regulated kinase (ERK) by the prothoracicotropic hormone in prothoracic gland cells and its developmental regulation in the silkworm, Bombyx mori. J Insect Physiol 2007:53:622-31.

[42] Lu YX, Denlinger DL, Xu WH. Polycomb repressive complex 2(PRC2) protein ESC regulates insect developmental timing by mediating $\mathrm{H} 3 \mathrm{~K} 27 \mathrm{me} 3$ and activating prothoracicotropic hormone gene expression. J Biol Chem 2013:288:23554-64

[43] Mané-Padróz D, Cruz J, Vilaplana L, Nieva C, Urena E, Belles X, et al. The hormonal pathway controlling cell death during metamorphosis in a hemimetabolous insect. Dev Biol 2010;346:150-60.

[44] Marchal E, Badisco L, Verlinden H, Vandersmissen T, Van Soest S, Van Wielendaele P, et al. Role of the Halloween genes, Spook and Phantom in ecdysteroidogenesis in the desert locust, Schistocerca gregaria. J Insect Physiol 2011:57:1240-8.

[45] Marchal E, Vandersmissen HP, Badisco L, Van de Velde S, Verlinden H, Iga M, et al. Control of ecdysteroidogenesis in prothoracic glands of insects: a review. Peptides 2010;31:506-19.

[46] Mizoguchi A, Ohsumi S, Kobayashi K, Okamoto N, Yamada N, Tateishi K, et al. Prothoracicotropic hormone acts as a neuroendocrine switch between pupal diapause and adult development. PloS ONE 2013;8:1-9.

[47] Mizoguchi A, Oka T, Kataoka H, Nagasawa H, Suzuki A, Ishizaki H. Immunohistochemical localization of prothoracicotropic hormone-producing neurosecretory cells in the brain of Bombyx mori. Dev Growth Differ 1990;32:591-8.

[48] Mohamed AAM, Wang QS, Bembenek J, Ichihara N, Hiragaki S, Suzuki T, et al. $\mathrm{N}$-acetyltransferase (nat) is a critical conjunct of photoperiodism between the circadian system and endocrine axis in Antheraea pernyi. PloS ONE 2014;9:1-14.

[49] Orrenius S, Zhivotovsky B, Nicotera P. Regulation of cell death: the calcium-apoptosis link. Nat Rev Mol Cell Biol 2003;4:552-65.

[50] Perez-Hedo M, Eizaguirre M, Sehnal F. Brain-independent development in the moth Sesamia nonagrioides. J Insect Physiol 2010;56:594-602.

[51] Perez-Hedo M, Pena RN, Sehnal F, Eizaguirre M. Gene encoding the prothoracicotropic hormone of a moth is expressed in the brain and gut. Gen Comp Endocrinol 2010:169:203-9.

[52] Pondeville E, Maria A, Jacques JC, Bourgouin C, Dauphin-Villemant C. Anopheles gambiae males produce and transfer the vitellogenic steroid hormone 20-hydroxyecdysone to females during mating. Proc Natl Acad Sci U S A 2008:105:19631-6.

[53] Pratt GE, Tobe SS. Juvenile hormones radiobiosynthesised by corpora allata of adult female locusts in vitro. Life Sci 1974;14:575-86.

[54] Rewitz KF, Yamanaka N, Gilbert LI, O'Connor MB. The insect neuropeptide PTTH activates receptor tyrosine kinase torso to initiate metamorphosis. Science 2009;326:1403-5.

[55] Roller H, Dahm KH. The chemistry and biology of juvenile hormone. Recent Prog Horm Res 1968;24:651-80.

[56] Rybczynski R, Gilbert LI. Protein kinase C modulates ecdysteroidogenesis in the prothoracic gland of the tobacco homworm, Manduca sexta. Mol Cell Endocrinol 2006;251:78-87.

[57] Rybczynski R, Snyder CA, Hartmann J, Gilbert LI, Sakurai S. Manduca sexta prothoracicotropic hormone: evidence for a role beyond steroidogenesis. Arch Insect Biochem Physiol 2009;70:217-29.

[58] Slama K. Insect hormones: more than 50 years after the discovery of juvenile hormone analogues (JHA, juvenoids). Terr Arthropod Rev 2013;6:1-77. 
G Model

PEP-69302; No. of Pages 10

10

A. De Loof et al. / Peptides $x x x$ (2014) $x x x-x x x$

[59] Llama K, Dukas J. Role of juvenile hormone in the hypermetabolic production of water revealed by the $0-2$ consumption and thermovision images of larvae of insects fed a diet of dry food. Eur J Entomol 2013;110:221-30.

[60] Stay B, Friedel T, Tobe SS, Mundall EC. Feedback control of juvenile hormove synthesis in cockroaches - possible role for ecdysterone. Science 1980;207:898-900.

[61] Stopple P, Briers T, Huybrechts R, Deloof A. Molting hormone, Juvenile hormone and the ultrastructure of the fat body of adult Sarcophagi bullate (Diptera). Cell Tissue Res 1981;221:233-44.

[62] Strehler EE, Treiman M. Calcium pumps of plasma membrane and cell interior. Cor Mol Med 2004;4:323-35.

[63] Tawfik AI, Mathova A, Sehnal F, Ismail SH. Haemolymph ecdysteroids in the coliteary and gregarious larvae of Schistocerca gregaria. Arch Insect Biochem Physio l 1996;31:427-38.

[64] Vafopoulou X, Steel CG. Synergistic induction of the clock protein PERIOD by insulin-like peptide and prothoracicotropic hormone in Rhodnius prolixus (Hemiptera): implications for convergence of hormone signaling pathways. Front Physio 2014;5:41.

[65] Van Mellaert H, De Loo A, Jurd L. Anti-juvenile hormone effects of newly described chemosterilants - benzyl-1,3-benzodioxoles and benzylphenols. Entomol Exp App 1983;33:83-8.

[66] Vandersmissen T [PhD dissertation] Neuropeptidergic Control of Ecdysteroidogenesis in Locusts. Leuven, Belgium: KU Leuven; 2008.

[67] Vandersmissen T, De Loo A, Gu SH. Both prothoracicotropic hormone and an autocrine factor are involved in control of prothoracic gland ecdysteroidogenisis in Locust migratoria and Schistocerca gregaria. Peptides 2007;28:44-50.
[68] Weaver RJ, Audsley N. Neuropeptide regulators of juvenile hormone synthesis structures, functions, distribution, and unanswered questions. Ann NY Accad Sci 2009;1163:316-29.

[69] Wei ZJ, Zhang QR, Kans L, Xu WH, Denlinger DL. Molecular characterizetimon and expression of prothoracicotropic hormone during development and pupal diapause in the cotton bollworm, Helicoverpa armiger. J Insect Physio 2005;51:691-700.

[70] Wikipedia: Thymosin. http://en.wikipedia.org/wiki/Thymosin [21.07.2014].

[71] Yamanaka N, Romero NM, Martin FA, Rewitz KF, Sun M, O'Connor MB, et al. Neuroendocrine control of Drosophila larval light preference. Science 2013;341:1113-6.

[72] Young SC, Yen WL, Gu SH. Transcriptional regulation of the PTTH receptor in prothoracic glands of the silkworm, Bombyx mort. J Insect Physiol 2012;58: 102-9.

[73] Chang Q, Denlinger DL. Molecular structure of the prothoracicotropic hormons gene in the northern house mosquito, Culex pipiens, and its expression analysis in association with diapause and blood feeding. Insect Mol Biol 2011;20:201-13.

[74] Chang QR, Denlinger DL. Dynamics of diapause hormone and prothoracicotropic hormone transcript expression at diapause termination in pupae of the corn earworm, Helicoverpa zeal. Peptides 2012;34: $120-6$.

[75] Zoller AL, Kersh GJ. Estrogen induces thymic atrophy by eliminating early thymic progenitors and inhibiting proliferation of beta-selected thymocytes. J Immunol 2006;176:7371-8.

Please cite this article in press as: De Loo A, et al. Initiation of metamorphosis and control of ecdysteroid biosynthesis in insects: The interplay of absence of Juvenile hormone, PTTH, and $\mathrm{Ca}^{2+}$-homeostasis. Peptides (2014), http://dx.doi.org/10.1016/j.peptides.2014.07.025 